\title{
Penicillin-Resistant Streptococcus Pneumoniae Meningitis With Subsequent Vertebral Osteomyelitis and Choledocholithiasis
}

\author{
Naoki Kasahata ${ }^{\mathrm{a}, \mathrm{b}}$, Naoaki Dan ${ }^{\mathrm{a}}$, Toshiyuki Yasui ${ }^{\mathrm{a}}$
}

\begin{abstract}
Penicillin-resistant Streptococcus pneumoniae (PRSP) meningitis is life threatening disease, and prevention and treatment of complication is important. We recently encountered two PRSP meningitis patients with undescribed complications: vertebral osteomyelitis and choledocholithiasis. Patient 1 was a 65 -year-old man who presented with PRSP meningitis, disseminated intravascular coagulation (DIC), infective endocarditis, vertebral osteomyelitis, and choledocholithiasis with gallstones. Patient 2 was a 57 -year-old man who presented with PRSP meningitis, DIC, acute renal failure, liver dysfunction, vertebral osteomyelitis, and choledocholithiasis with gallstones. Diffusion-weighted brain MRI of both patients revealed high-intensity areas in the posterior horns of the lateral ventricles consistent with ventriculitis. Spinal MRI revealed low-intensity on T1-weighted images and high-intensity on T2-weighted images of the lumbar vertebral bodies and intervertebral disks, consistent with vertebral osteomyelitis. Technetium-99m bone scans revealed accumulation in the lumbar vertebrae. Computed tomography (CT) scans on admission revealed no gallstones, whereas CT scans after abdominal pain or liver dysfunction revealed choledocholithiasis and gallstones. Vertebral osteomyelitis and choledocholithiasis should be noted as possible complications of PRSP meningitis.
\end{abstract}

Keywords: Penicillin-resistant Streptococcus pneumoniae; Meningitis; Vertebral osteomyelitis; Choledocholithiasis; Gallstones

\section{Introduction}

Bacterial meningitis is a serious and life-threatening disease,

Manuscript accepted for publication October 31, 2013

${ }^{a}$ Department of Medicine, Tokyo Metropolitan Ohtsuka Hospital, Tokyo, Japan

${ }^{\mathrm{b}}$ Corresponding author: Naoki Kasahata, Department of Medicine, Division of Neurology, Tokyo Metropolitan Ohtsuka Hospital, 2-8-1 Minamiohtsuka, Toshima-ku, Tokyo 170-8476, Japan.

Email: n_kasa-o@ohtsuka-hospital.toshima.tokyo.jp

doi: http://dx.doi.org/10.14740/jmc1598e with an overall mortality rate of $21 \%$ [1]. The most common pathogen of community-acquired acute bacterial meningitis is Streptococcus pneumoniae (51\% of episodes), which has a mortality rate of $30 \%$ [1]. The epidemiology of bacterial meningitis has recently changed [1].

Vertebral osteomyelitis has been in most patients a secondary complication of a distant infection with haematogenous seeding, and it may be complicated by direct seeding in different compartments, resulting in paravertebral, epidural, or psoas abscess [2-4]. Magnetic resonance imaging (MRI) is highly accurate (90\%) in diagnosing spinal osteomyelitis [3]. Technetium-99m bone scans are typically positive within a few days after the onset of symptoms, though their reported accuracy in detecting vertebral osteomyelitis is 67\% [3]. Vertebral osteomyelitis is a rare complication of bacterial meningitis [5]. Previous authors have reported some patients with bacterial meningitis and vertebral osteomyelitis [2, 5-10].

We recently encountered two PRSP meningitis patients with vertebral osteomyelitis and choledocholithiasis. Herein we report two patients of PRSP meningitis with undescribed complications: vertebral osteomyelitis and choledocholithiasis.

\section{Case Report}

This study is equivalent to be approved by an appropriate institutional committee on human research (the ethics committee of Tokyo Metropolitan Ohtsuka Hospital).

\section{Case 1}

A 65-year-old man admitted to the hospital because of headache, vomiting, and fever.

The patient had been well until 2 days before admission, when he experienced low back pain after working with heavy objects. Fever increased to $38^{\circ} \mathrm{C}$. At night, he complained of headache 15 minutes after he began to vomit.

He worked as an executive, very hard without holidays. He always slept approximately 5 hours.

On examination, he was disoriented and was able to lo- 

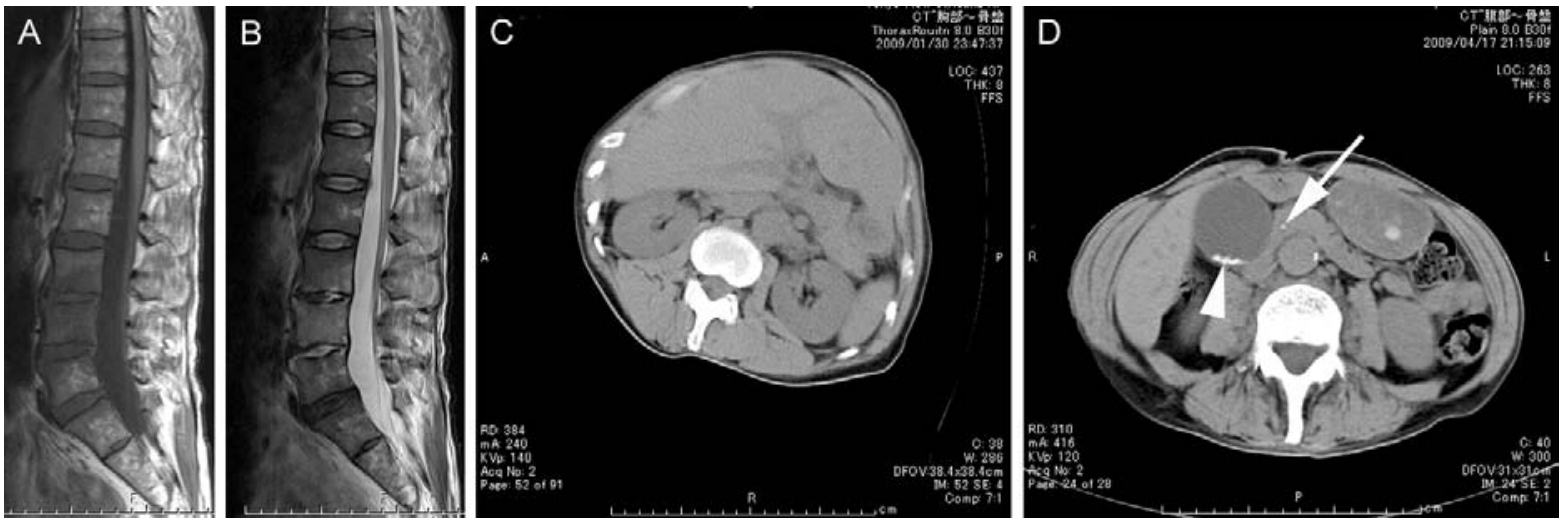

Figure 1. Magnetic resonance imaging (MRI) of lumbar vertebrae and abdominal CT scan in patient 1 . Vertebral osteomyelitis and choledocholithiasis with gall stones. A: T1-weighted image (FA 150 TR 600 TE 12) of the lumbar vertebrae shows regions of low intensity in the L4 vertebral body and L3-4 and L4-5 intervertebral discs. B: Fat-suppression T2-weighted image (FA 150 TR 4000 TE 96) of the lumbar vertebrae shows regions of high intensity in the L3, L4, and L5 vertebral bodies and L3-4, L4-5 intervertebral discs. C: No gallstones or choledocholithiasis on admission. D: A stone in the lower portion of the common bile duct (arrow) and small stones in the gall bladder (arrowhead) on the 77th hospital day.

calize painful stimuli on his extremities. Seizures of his right arm were sometimes observed. Deep tendon reflexes were decreased and Achilles tendon reflexes were absent. The patient exhibited bilateral Babinski signs. The patient exhibited nuchal rigidity without Kernig sign. The remainder of the examination was normal.

The cerebrospinal fluid (CSF) exhibited pleocytosis, with cells $=557.3$ per milliliter, mononuclear cells: polymorphonuclear cells $=30.6: 69.4$, protein $378 \mathrm{mg} / \mathrm{dL}$, glucose $0 \mathrm{mg} / \mathrm{dL}$. Laboratory examination yielded the following findings: WBC $19,400 / \mu \mathrm{L}$ (neutrophils $94.3 \%$, band forms $12.5 \%$ ), platelets $81,000 / \mu \mathrm{L}$, FDP $90 \mu \mathrm{g} / \mathrm{mL}$ D-dimer 15.4 $\mu \mathrm{g} / \mathrm{mL}$, albumin $2.3 \mathrm{~g} / \mathrm{dL}$, ALP $921 \mathrm{U} / \mathrm{L}, \gamma \mathrm{GTP} 124 \mathrm{U} / \mathrm{L}$, CRP $29.52 \mathrm{mg} / \mathrm{dL}$. CSF and blood gram staining revealed Gram-positive cocci and cultures grew penicillin-resistant
Streptococcus pneumoniae (PRSP). Results of other laboratory tests were normal. An electroencephalogram revealed $\beta$-activity with $\delta$ waves and spikes. Diffusion-weighted MRI of the brain revealed high intensity in posterior horns of the lateral ventricles consistent with ventriculitis.

Cefotaxime $2 \mathrm{~g} 4$ times/day and meropenem $2 \mathrm{~g} 3$ times/ day were initiated. Dexamethasone $8 \mathrm{mg} 3$ times/day for 3 days, gabexate mesylate $2,000 \mathrm{mg} /$ day, Phenytoin $500 \mathrm{mg} /$ day, and Glycerol $400 \mathrm{~mL} /$ day were administered. These treatments improved the bacterial meningitis. On the third hospital day, a grade 3 high-pitched systolic murmur was heard at the apex. On the fourth hospital day, he complained of low back pain. On the 10th hospital day, echocardiography revealed vegetation on the mitral valve, and infective endocarditis was diagnosed. On the 18th hospital day, fever
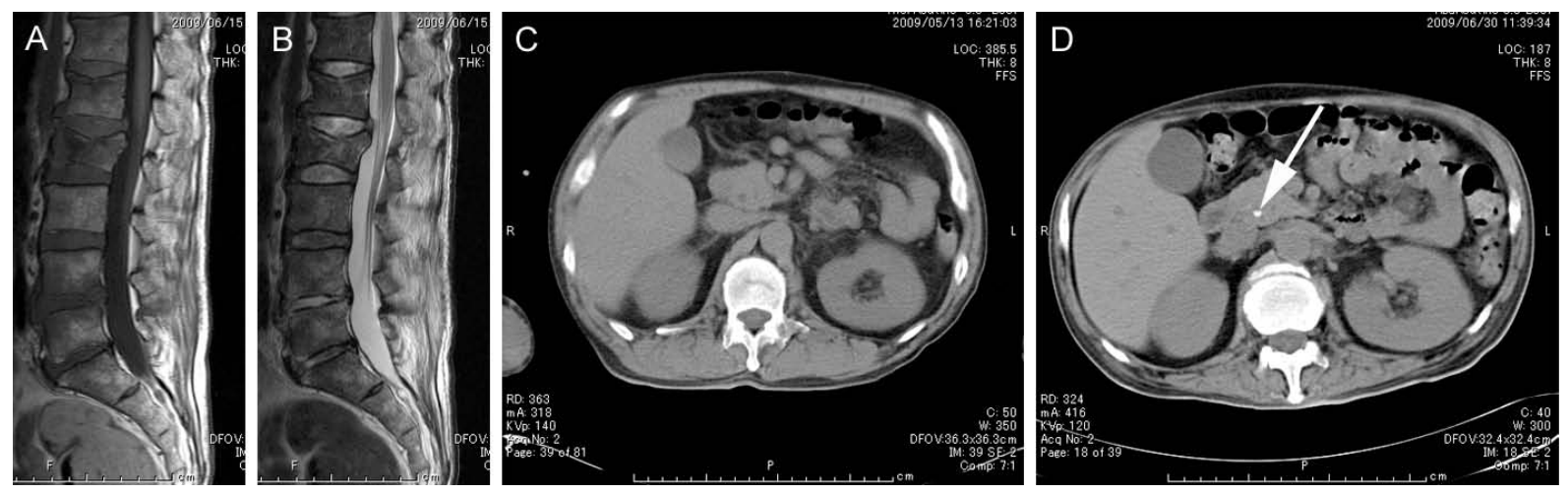

Figure 2. MRI of lumbar vertebrae and abdominal CT scan in patient 2. Vertebral osteomyelitis and choledocholithiasis with gall stones. A: T1-weighted image (FA 150 TR 600 TE 12) shows regions of low intensity in the L4 and L5 vertebral bodies and L4-5 intervertebral disc. B: T2-weighted image (FA 150 TR 4000 TE 96) shows regions of high intensity in the L4 and L5 vertebral bodies and L4-5 intervertebral disc. C: No gallstones or choledocholithiasis on admission. D: A stone in the lower portion of the common bile duct (arrow) on the 50th hospital day. 
Table 1. Characteristics of Patients With Meningitis and Vertebral Osteomyelitis

\begin{tabular}{|c|c|c|c|c|c|}
\hline Author & $\begin{array}{l}\text { Age/ } \\
\text { sex }\end{array}$ & Bacteria & $\begin{array}{l}\text { Gall } \\
\text { stone }\end{array}$ & Choledocholithiasis & Characteristics \\
\hline Markus et al Case 1 [6] & $60 / \mathrm{M}$ & SA & N/A & N/A & \\
\hline Case 2 & $46 / F$ & SA & $(-)$ & $(-)$ & A psoas abscess \\
\hline Chemlal et al [2] & $52 / \mathrm{M}$ & PRSP & $(-)$ & $(-)$ & \\
\hline Turner et al patient $8[10]$ & $79 / \mathrm{F}$ & PSSP & N/A & N/A & Endocarditis \\
\hline Poyanli et al [8] & $51 / \mathrm{F}$ & PSSP & $(-)$ & $(-)$ & Psoas abscesses \\
\hline Brouwer et al Case 1 [5] & $51 / \mathrm{M}$ & PSSP & N/A & N/A & \\
\hline Case 2 & $39 / \mathrm{F}$ & PSSP & N/A & $\mathrm{N} / \mathrm{A}$ & \\
\hline Fukushima et al [6] & $62 / \mathrm{M}$ & Group B S & $\mathrm{N} / \mathrm{A}$ & N/A & Epidural abscess \\
\hline Tsutsumi et al [9] & $62 / \mathrm{M}$ & Group B S & $(-)$ & $(-)$ & $\begin{array}{l}\text { Rectal ulcer Paraspinal } \\
\text { muscle abscess }\end{array}$ \\
\hline Patient 1 & $65 / \mathrm{M}$ & PRSP & $(+)$ & $(+)$ & Endocarditis \\
\hline Patient 2 & $57 / \mathrm{M}$ & PRSP & $(+)$ & $(+)$ & \\
\hline
\end{tabular}

SA: Staphylococcus aureus; N/A: not available; PRSP: penicillin resistant Streptococcus pneumoniae; PSSP: penicillin sensitive Streptococcus pneumoniae; S: Streptococcus

gradually rose to $38{ }^{\circ} \mathrm{C}$. Gentamicin $65 \mathrm{mg} 3$ times/day and vancomycin $0.9 \mathrm{~g}$ twice/day were initiated. Spinal MRI in patient 1 revealed low intensity areas on T1-weighted images and high intensity areas on T2-weighted images of the L4 vertebral body and low intensity areas on $\mathrm{T} 1$-weighted images of the L3/4 and L4/5 intervertebral disc spaces (Fig. 1A, B). Technetium-99m bone scans in patient 1 showed accumulations in L4 and L5. The patient's low back pain was improved. Ceftriaxone $2 \mathrm{~g}$ /day was added on the 40th hospital day because of exacerbated infective endocarditis. On the 65th hospital day, the patient complained of abdominal pain. Sulbactam/cefoperazone $2 \mathrm{~g}$ /day was administered and the abdominal pain improved. CT scan on admission in patient 1 revealed no gallstones (Fig. 1C), while after abdominal pain it revealed choledocholithiasis and gallstones (Fig. 1D).

\section{Case 2}

A 57-year-old man admitted to the hospital because of headache.

The patient had been well until 2 days before admission, when he complained of chills and fever. Five days before admission, he had gone on a trip for his work without sleep. Three days before admission, he returned to Tokyo and worked, and returned home at midnight. He had watery diar- rhea at that time. One day before admission, he vomited and had a fever of $40.5^{\circ} \mathrm{C}$. The day of admission, he complained of severe headache and loss of appetite and vomited.

He drank half a bottle of shouchu (Japanese spirits) per day. He was a 57-year-old singer who worked night and slept from 5:00 A.M. to 4:00 P.M. daily.

On examination, the patient was already sedated and disturbance of consciousness was unable to be accurately evaluated. Partial seizures within a minute duration were sometimes observed. Deep tendon reflexes were decreased in the upper extremities. The patient exhibited nuchal rigidity with a questionable Kernig sign. The remainder of the examination was normal.

The CSF exhibited pleocytosis: cells $=116.7$ per milliliter, all cells were polymorphonuclear cells, protein 284 $\mathrm{mg} / \mathrm{dL}$, glucose $4 \mathrm{mg} / \mathrm{dL}$. Laboratory examination yielded the following findings: WBC $10,000 / \mu \mathrm{L}$ (myelocytes $1.0 \%$, metamyelocytes $4.0 \%$, band forms $38.0 \%$, neutrophils $43.0 \%$ ), platelets $117,000 / \mu \mathrm{L}$, FDP $90.0 \mu \mathrm{g} / \mathrm{mL}$, D-dimer $70.0 \mu \mathrm{g} / \mathrm{mL}$, BUN $38.8 \mathrm{mg} / \mathrm{dL}$, creatinine $2.7 \mathrm{mg} / \mathrm{dL}, \mathrm{K} 3.0$ $\mathrm{mEq} / \mathrm{L}$, AST $236 \mathrm{U} / \mathrm{L}$, ALT $110 \mathrm{U} / \mathrm{L}, \mathrm{LDH} 512 \mathrm{U} / \mathrm{L}, \gamma \mathrm{GTP}$ $146 \mathrm{U} / \mathrm{L}$, blood glucose $195 \mathrm{mg} / \mathrm{dL}$, CRP $62.56 \mathrm{mg} / \mathrm{dL}$. Results of CSF Gram staining were suggestive of pneumococci, and cultures grew PRSP. Results of other laboratory tests were normal. Results of urinalysis included $3+$ occult 
blood, 3+ protein, RBC 20-29/HPF (high power field), and white cell casts $20-29 / \mathrm{F}$. Electroencephalogram revealed $\beta$ activity and some spikes. Findings of brain MRI suggested ventriculitis.

Meropenem 2 g 3 times/day and Dexamethasone $8 \mathrm{mg} 4$ times /day for 3 days were initiated. Oliguria and DIC were observed (platelets were $57,000 / \mu \mathrm{L}$ on the second hospital day). Fluid, low-dose dopamine, heparin, and gabexate mesylate were injected. From the ninth hospital day, he exhibited rash. He was diagnosed with erythema multiforme, which was suspected to be drug-induced. Meropenem was discontinued on the 10th hospital day and vancomycin $0.5 \mathrm{~g}$ 4 times/day, ceftriaxone $2 \mathrm{~g}$ twice/day, and micafungin 100 $\mathrm{mg}$ /day were administered. CSF findings were normalized and antibiotics were discontinued on the 31 st hospital day and liver dysfunction was improved. However, he exhibited fever and low back pain. Ceftriaxone $2 \mathrm{~g}$ once/day was injected on the 34th hospital day. Spinal MRI in patient 2 revealed low intensity areas on T1-weighted images and high intensity areas on T2-weighted images of the L4 and L5 vertebral bodies and L4/5 intervertebral disc space (Fig. 2A, B). Technetium-99m bone scans in both patient 2 showed accumulations in L4 and L5.

On the 62nd hospital day, he suddenly exhibited liver dysfunction. CT scan on admission in patient 2 revealed no gallstones (Fig. 2C), while after liver dysfunction it revealed choledocholithiasis and gallstones (Fig. 2 D).

\section{Discussion}

The characteristics of these patients include the following: patient 1 was a 65-year-old executive who worked hard without holidays and slept very little. He showed PRSP meningitis, DIC, infective endocarditis, vertebral osteomyelitis and choledocholithiasis with gallstones. Patient 2 was a 57-year-old singer who worked night and slept from 5:00 A.M. to 4:00 P.M. daily. He was a heavy drinker, and traveled and worked without sleep before the onset of meningitis. He showed PRSP meningitis, DIC, acute renal failure, liver dysfunction, vertebral osteomyelitis, and choledocholithiasis with gallstones. Similarities in background included hard work at night and little sleep before onset. Fatigue and stress predisposed Pneumococcal infection.

We think of vertebral osteomyelitis as a complication of PRSP meningitis. Consequently, PRSP meningitis can complicate vertebral osteomyelitis according to the findings of the present case report. Previous authors reported patients of vertebral osteomyelitis complicating pneumococcal meningitis (Table 1) [2, 3, 5, 9, 10]. Hematogenous seeding from the CSF space to lumbar vertebrae may cause vertebral osteomyelitis. We think of vertebral osteomyelitis as a possible complication of bacterial meningitis, especially that due to PRSP.
The question arises whether the choledocholithiasis in these two patients was a complication of vertebral osteomyelitis or PRSP meningitis. There is no evidence for this, and there has been no similar case report. The gallstones were very small, and were not present at disease onset. Therefore, these gall stones were formed after disease onset. There may be an association between choledocholithiasis and PRSP meningitis with vertebral osteomyelitis. Increase in local blood calcium concentration by vertebral osteomyelitis may result in formation of gallstones and choledocholithiasis. Weight loss and gallbladder hypomotility due to parenteral nutrition predisposed cholesterol stones. We think of choledocholithiasis as a possible complication of PRSP meningitis or vertebral osteomyelitis.

We have described two patients of PRSP meningitis with vertebral osteomyelitis and choledocholithiasis. Vertebral osteomyelitis and choledocholithiasis should be noted as possible complications of PRSP meningitis.

\section{Disclosure}

There are no financial interests tied to this original research, neither is there any other side support.

\section{Grant Support}

None.

\section{References}

1. van de Beek D, de Gans J, Spanjaard L, Weisfelt M, Reitsma JB, Vermeulen M. Clinical features and prognostic factors in adults with bacterial meningitis. N Engl J Med. 2004;351(18):1849-1859.

2. Chemlal K, Trouillet JL, Carbon C, Yeni P. Vertebral osteomyelitis and meningitis due to a penicillin-resistant pneumococcal strain. Eur J Clin Microbiol Infect Dis. 1996;15(11):893-895.

3. Zimmerli W. Clinical practice. Vertebral osteomyelitis. N Engl J Med. 2010;362(11):1022-1029.

4. Lew DP, Waldvogel FA. Osteomyelitis. Lancet. 2004;364(9431):369-379.

5. Brouwer MC, de Gans J, Heckenberg SG, Kuiper H, van Lieshout HB, van de Beek D. Vertebral osteomyelitis complicating pneumococcal meningitis. Neurology. 2008;71(8):612-613.

6. Fukushima K, Fushimi T, Yamamoto K, Morita H, Yoshida K, Matsuda M, Ikeda S. Pictures in clinical medicine. Spinal epidural abscess with osteomyelitis as a cause of bacterial meningitis. Intern Med. 2008;47(9):883. 
7. Markus HS, Allison SP. Staphylococcus aureus meningitis from osteomyelitis of the spine. Postgrad Med J. 1989;65(770):941-942.

8. Poyanli A, Poyanli O, Akan K, Sencer S. Pneumococcal vertebral osteomyelitis: a unique case with atypical clinical course. Spine (Phila Pa 1976). 2001;26(21):23972399.

9. Tsutsumi R, Saito M, Yoshizawa T. [Group B streptococ- cus meningitis and infection surrounding the spinal canal caused by bacterial transmission from rectal ulcer via Batson's plexus]. Rinsho Shinkeigaku. 2011;51(7):493498.

10. Turner DP, Weston VC, Ispahani P. Streptococcus pneumoniae spinal infection in Nottingham, United Kingdom: not a rare event. Clin Infect Dis. 1999;28(4):873881. 